
\title{
Reseserch Soure \\ Feasibility of The Use of Neutrophil To Lymphocyte Ratio (NLR) As An Adjunct To Cytology To Suggest Thyroid Malignancy: A Prospective Study
}

\section{Pawan Kumar Tiwari}

Armed Forces Medical College

Biju Varghese

Armed Forces Medical College

Arunjeet KK

Armed Forces Medical College

Pankaj P Rao

Armed Forces Medical College

Dronacharya Routh ( $\nabla$ dronacharya72@gmail.com )

Armed Forces Medical College https://orcid.org/0000-0002-9202-1700

\section{Research Article}

Keywords: Neutrophil Lymphocyte Ratio, Thyroid Malignancy, Cytology, Sensitivity, Specificity

Posted Date: July 15th, 2021

DOl: https://doi.org/10.21203/rs.3.rs-539030/v1

License: (9) This work is licensed under a Creative Commons Attribution 4.0 International License.

Read Full License 


\section{Abstract}

Background: Inflammation is known to be associated with cancer development and progression. NLR is a simple and efficient marker of inflammation in numerous malignancies.

Aim: The study aimed to investigate usefulness of neutrophil to lymphocyte ratio (NLR) as an adjunct to Fine needle aspiration cytology (FNAC) to suggest thyroid malignancy confirmed by histopathological report post operatively in Bethesda class III, IV \& V.

Methods: Prospective observational study at a tertiary care hospital over 2 years.

Results: NLR in the benign group and malignant group were 1.75 \pm 0.23 and $2.19 \pm 0.29$ (mean \pm SD) respectively ( $p$-value $<0.001$ ). Area under ROC curve plotted was 0.823 ( $p$-value $<0.001$ ), with recommended cut-off of 1.9 . Relatively high sensitivity and specificity at $87.5 \%$ and $82.5 \%$ implies excellent diagnostic accuracy.

Conclusion: NLR with a value of 1.9 or higher is recommended as predictor of malignancy in thyroid nodule.

\section{Introduction}

Thyroid nodules are common in the general population with prevalence rates varying with the method of discovery; $2-6 \%$ by palpation, $19-35 \%$ by ultrasonography and $8-65 \%$ on autopsy [1]. While the vast majority of these lesions are benign, approximately $4-6.5 \%$ of all palpable nodules have been found to be malignant [2]. The diagnosis of thyroid nodule is being made with increasing frequency in recent years due to better availability of sensitive imaging techniques [3]. Since these nodules are common, their clinical significance lies in their functional status, their potential to cause pressure symptoms and most importantly to exclude malignancy.

Carcinoma of the thyroid gland constitutes almost $90 \%$ of all endocrine malignancies with an estimated annual incidence of 122,800 cases worldwide [4]. It is one of the most curable cancers, however the differential diagnosis can often be elusive. The fine-needle aspiration cytology (FNAC) is the most useful preoperative diagnostic tool in the differential diagnosis of the thyroid nodules, however $15-30 \%$ of FNACs (Bethesda III, IV) usually fall into the indeterminate category due to overlapping morphologic features on cyto-pathological review and is confirmed to be benign or malignant only after post-operative histopathological examination (HPE) report. Since the surgical management of a benign compared with a malignant thyroid nodule differs substantially, additional markers are required to be identified to improve the accuracy of the diagnosis of malignancy and optimize and improve the clinical care of patients presenting with suspicious thyroid lesions. There are certain additional findings such as the patient's age, gender, size of the nodule or features on ultrasound which are known to be associated with malignancy. However, specificity of these features is too low to be clinically useful [5]. 
The inflammation process and immune dysfunctions as a predisposing factor for the development of malignancy was first noted by Virchow [6]. The presence of leukocytes with macrophages, mast cells, and T cells is characteristically found around the inflammation surrounding the neoplastic tissues [6]. Multiple studies have provided increasing and consistent evidence that the inflammation is related to the cancer development and progression [7]. Its role has been documented in such complex as cancer development, tumour progression, angiogenesis and metastasis [8]. Hence, systemic inflammatory markers such as C-reactive protein (CRP), albumin concentration and neutrophil to lymphocyte ratio (NLR) were investigated and shown to be independent prognostic factors in the cancer patients [9]. The neutrophil-lymphocyte ratio (NLR) has been found to be associated with the progression and the survival of most cancers [10,11]. Increased NLR levels are a bad prognostic marker for various cancers [12]. Use of NLR is a fast, cheap, easily detectable method and is frequently utilised in daily practice. NLR is not expected to contribute to the Bethesda category II and VI lesions where sensitivity and specificity of FNAC is very high. Sensitivity and specificity of FNAC is brought down by indeterminate category of lesions i.e Bethesda III \& IV. This study aims to investigate the NLR levels in Benign and Malignant thyroid nodules confirmed by post-operative HPE report which can further act as an adjunct to predict malignancy in Bethesda III, IV, V thyroid nodules.

\section{Materials And Methods}

This observational study is an ongoing study conducted in a tertiary care teaching hospital and currently the data for a period of two years is being analysed. All the patients undergoing elective surgery for nodular thyroid goitre with FNAC Bethesda III, IV \& V in this center during this period were recruited in the study after taking written informed consent. Permission for the study was granted from the Institutional Ethics Committee. Bethesda class II \& VI were not considered as sensitivity and specificity of FNAC is very high. Patients with thyroid enlargements due to other causes like thyroiditis and those with systemic diseases such as active or chronic infections, diabetes mellitus, autoimmune disease, renal and liver disease were excluded. Similarly, patients on anticoagulants including aspirin, steroids or h/o alcohol intake and smoking were not included.

Sample size calculation: A total of 15 patients of each group were recruited in the pilot study for sample size calculations. Mean NLR $(\mu 1)$ was calculated for patients with benign nodule. Similarly mean NLR $(\mu 2)$ was calculated for other group of patients that is with malignant nodules based on the postoperative histopathology report. Standard deviation SD1 and SD2 was calculated for each mean NLR. From pilot data of patients $\mu 1=2.21, \mu 2=1.86, \mathrm{SD} 1=0.58, \mathrm{SD} 2=0.52$. Taking a as $1 \%$ and power as $80 \%$, the required sample came out as 38 patients each for Benign thyroid Nodule and Malignant Thyroid Nodule. Hence, a sample size of 40 in each group was decided.

Method: All the patients undergoing elective surgery for nodular thyroid goitre with FNAC III, IV, V in this center during this period were recruited in the study after written informed consent. The phlebotomy samples for hemoglobin, complete blood counts, absolute neutrophil count, absolute lymphocyte count, differential leucocyte count, TSH and free $\mathrm{T}_{4}$ were collected and analyzed in the hospital laboratory. The 
blood samples were collected in the admission ward itself by the study surgical resident in a single prick phlebotomy on the day of surgery between 0800 to 0900 hours. At least $4 \mathrm{ml}$ of blood samples were collected for all patients in a EDTA bottle for evaluation of complete blood counts, absolute neutrophil count, absolute lymphocyte count and differential leucocyte count. Samples in sterile bottles with at least $4 \mathrm{ml}$ in the bottle were collected for $\mathrm{TSH}$ and free $\mathrm{T}_{4}$. The automatic hematology analyzer Beckman Coulter Hematology Analyzer LH 750 (Beckman Coulter Inc., CA, USA) was used to assess the blood counts. The levels of TSH and free $\mathrm{T}_{4}$ were obtained by Beckman Coulter DX1800 Immunoassay Analyser (Beckman Coulter Inc., CA, USA).

The NLR was calculated by dividing absolute neutrophil count by absolute lymphocyte count and the values obtained were recorded and plotted on a graph. The final diagnosis was confirmed in the postoperative final pathology sample. The Patients were grouped in two different groups Group A with benign HPE and Group B with malignant HPE results. NLR values in both groups were collected and analysed. The patient groups were compared with regards to the age of patients, gender, thyroid function tests, neutrophil, lymphocyte, and NLR. The primary end point for the study was evaluation of pre-operative NLR correlation with thyroid carcinoma and establishing the range of NLR values in benign and malignant nodule in Nodular goitre. In this study we had recruited 107 patients with thyroid nodule (Bethesda III,IV,V). We recruited more patients as we required 40 in each group. Remaining 27 patients were excluded for this study, however will be recruiting them later for further subgroup analysis as this is an ongoing study.

Data analysis: The data generated noted on a pre-designed proforma was entered in a excel sheet to statistically analyze the set using the SPSS Ver. 22 software (IBM Inc.). The numerical variables were expressed as mean \pm standard deviation (SD). Categorical variables were presented as absolute values or percentage/ proportions. The two-sample t-test (Student's t) was used for analyzing the quantitative variables with normal distribution. The Chi $\left(\chi^{2}\right)$ square test was used for skewed distribution and for categorical variables. In comparisons using Student's t test, $80 \%$ confidence intervals for the mean difference in response provided a range of likely values to assess clinical significance. Receiver operating characteristics (ROC) curve analysis was used to define optimal cut-offs of NLR, for which specificities, positive and negative predictive values (PPV, NPV) and overall accuracies were calculated. For all tests of significance, $p$-values less than 0.05 were considered statistically significant.

\section{Results}

The flowchart (Fig. 1) demonstrates the flow of participants in this study. The characteristics (age, gender, male/female ratio, TSH levels and leucocyte counts) of the patients who were eligible to be enrolled and who participated in the study were comparable in both benign and malignant group (Table.1). The difference of neutrophil to lymphocyte ratios between the benign and malignant was noted to be highly significant. The respective values for mean and standard deviation for benign group were 1.75 and 0.23 whereas these were 2.19 and 0.29 for the malignant group (Fig. 2). The estimated p-value comparing the mean and SD was found to be $<0.001$. 
NLR value of 1.9 was calculated by receiver operating curve (ROC) analysis (Fig. 3) as an optimal cut-off value to discriminate between benign and malignant nodules. ROC was plotted where smallest cut-off value is the minimum observed test value minus 1 , and the largest cut-off value is the maximum observed test value plus 1 . All the other cut-off values are the averages of two consecutive ordered observed test values. The cut-off ( $<1.9)$ of Neutrophil to Lymphocyte ratio (NLR) by ROC which is highlighted by dotted line on ROC has relatively high sensitivity and specificity in predicting the prevalence of malignancy. The area under the ROC curve was 0.823 with $p$-value of $<0.001$.

The distribution graphs (Fig. 4) of the patients also show the difference between the benign and malignant categories of patients with the best estimated cut-off value calculated at NLR of 1.9 according to the ROC curve. The difference of neutrophil to lymphocyte ratios between the benign and malignant as plotted on ROC revealed the sensitivity and specificity of $87.5 \%$ and $82.5 \%$ which implies excellent diagnostic accuracy (Table 2).

Table 1

Preoperative demographic data of both the groups.

\begin{tabular}{|llll|}
\hline Characteristics & $\begin{array}{l}\text { Benign group } \\
\text { (Mean } \pm \text { SD) }\end{array}$ & $\begin{array}{l}\text { Malignant group } \\
\text { (Mean } \pm \text { SD) }\end{array}$ & p-value \\
\hline Age & $44.725 \pm 12.22$ & $42.35 \pm 12.69$ & 0.39 \\
\hline M: F Ratio & 0.66 & 0.82 & 0.61 \\
\hline TSH levels & $1.68 \pm 0.67$ & $1.64 \pm 0.63$ & 0.78 \\
\hline TLC levels & $7666.25 \pm 1157.77$ & $7755 \pm 1107.65$ & 0.72 \\
\hline
\end{tabular}

Table 2

Diagnostic efficacy indices calculated with ROC curve.

\begin{tabular}{|llll|}
\hline Indices & Value & $\mathbf{9 5 \%} \mathbf{C l}$ \\
\cline { 3 - 4 } & & Lower Limit & Upper Limit \\
\hline Sensitivity & 0.875 & 0.732 & 0.958 \\
\hline Specificity & 0.825 & 0.672 & 0.927 \\
\hline Positive Predictive Value & 0.833 & 0.685 & 0.942 \\
\hline Negative Predictive Value & 0.868 & 0.72 & 0.946 \\
\hline Positive Likelihood Ratio & 5.000 & 2.526 & 9.899 \\
\hline Negative Likelihood Ratio & 0.152 & 0.066 & 0.348 \\
\hline
\end{tabular}

\section{Discussion}


Inflammation has been innately associated with the development of many cancers and is involved in almost all of the pathogenic steps of malignancy initiation and propagation including its dissemination. Neutrophils have been implicated in directly or indirectly inducing tumor growth [13-16]. An elevated ratio of peripheral neutrophils-to-lymphocytes (NLR) is the simplest marker of systemic inflammatory response and has been recently recognized as an independent risk factor in the diagnosis of tumor development and a poor prognostic marker of many cancers

NLR has been incorporated in a simple score for men with metastatic castration- resistant prostate cancer. Some smaller studies report that chemotherapy can normalize elevated NLR early after the introduction of treatment and that patients with normalized NLR may have improved outcome [17]. Similarly, in women increased pretreatment NLR values was predictive of survival in ovarian cancer [18]. There was a direct correlation found in between tumor stage of gastro-esophageal carcinomas and NLR values in one Japanese study [19]. In colorectal cancers, NLR was associated with both predictive prognostic value as well as survival rates [20]. Same effects were also noted in hepatocellular carcinomas with added correlation with tumor invasion, occurrence of nodal disease and satellite lesions [21]. Poor prognostic markers such as weight loss, reduced functional capacity and poor performance status in pancreatic malignancies and survival in patients with lung cancer were likewise associated with increased NLR values $[22,23]$.

As it is inexpensive easily calculated and readily available from routine blood tests, its use in the preoperative assessment and postoperative follow-up of malignant thyroid cancer patients has been proposed but remains as a matter of debate due to mixed outcomes in comparison studies. A study by Kocer et al in 2015 compared the NLR values in papillary thyroid cancer, lymphocytic thyroiditis and benign disease and found higher values in patients with malignancy [24]. In a similar fashion, Seretis et al also reported higher NLR levels in papillary cancer as compared to benign thyroid disease [25]. In the same way Liu et al and Lang et al noticed NLR to be correlated with diameter of the lesion and the stage [26]. Manatakis et al. noted that higher NLR levels were seen in more aggressive tumours exhibiting features of extrathyroidal invasion, lymph node metastasis, bilaterality, multifocality and poor histopathological type [26]. A review of forty thousand patients with solid tumours done by Templeton et al. demonstrated that high NLR was associated with worse survival [26]. In contrast, Soyder et al found no difference in NLR values when studying follicular neoplasia. Likewise, Cho et al also could not demonstrate any difference of NLR values in benign and malignant disease [26].

This study proved that the difference between preoperative NLR values did correlate very significantly ( $p$ value $<0.001$ ) with malignancy and thus could be used to prognosticate the risk of malignancy. These results were similar to the studies reported by Kim et al. who noted high NLR to be associated with malignant disease, tumour diameter more than $1 \mathrm{~cm}$ and lympho-vascular invasion [27]. In a similar fashion, Gong et al. also reported association of high NLR with tumour diameter, lymph node metastasis and multifocality [28]. This study also adds credence to the findings of Seretis et al, Kocer et al, Liu et al, Lang et al and Manatakis et al. by providing evidence of trend of higher NLR subsets in the group of 
patients with thyroid malignancies. The added advantage of the ease of performing the score also adds to the usefulness of this test.

The limitations of this study include the small size of the study population. Validating the findings in larger trials can add to the veracity of results. As it is an ongoing study, further correlation of NLR with TNM staging in malignant cases and correlation among the various malignant thyroid disorders and other clinical-pathological parameters with the corresponding NLR values will be further published. The authors feel that a larger, well designed study can be done to look for association of NLR with other variables and obtain specific cut-off values to indicate malignancy in thyroid nodules.

\section{Conclusion}

Neutrophil to Lymphocyte Ratio (NLR) a simple, ubiquitous, universally available screening modality can be utilised to assist in differentiating between benign and malignant nodules in multinodular goitre. This study studied forty patients each belonging to either group in post-operate period and found the NLR to be $1.75 \pm 0.23$ and $2.19 \pm 0.29$ (mean $\pm S D$ ) in the benign and malignant groups with a highly significant p-value of $<0.001$.

The ROC curve plotted for the test revealed the cut-off of 1.9 for Neutrophil to Lymphocyte ratio (NLR) to differentiate between benign and malignant nodules in goitrous conditions. It has relatively high sensitivity and specificity at $87.5 \%$ and $82.5 \%$ which implies excellent diagnostic accuracy. Thus, it can be used to suggest benign or malignant nature prior to surgery and adds to the decision-making process.

\section{Declarations}

Funding: No funding sources

Conflicts of interest/Competing interests: None declared

Availability of data and material: Available

Ethical approval: The study was approved by the Institutional Ethics Committee.

Authors' contributions: All authors have contributed to the concept, analysis, writing and modification of the manuscript.

Consent to participate and publication: Informed consent was obtained from all individual participants included in the study. Patients signed informed consent regarding publishing their data.

Code availability: NA

\section{References}


1. Dean DS, Gharib H. Epidemiology of thyroid nodules. Best Pract Res Clin Endocrinol Metab. 2008;22(6):901-11.

2. Lin JD, Chao TC, Huang BY, Chen ST, Chang HY, Hsueh C. Thyroid cancer in the thyroid nodules evaluated by ultrasonography and fine-needle aspiration cytology. Thyroid. 2005;15(7):708-17.

3. Li N, Du XL, Reitzel LR, Xu L, Sturgis EM. Impact of enhanced detection on the increase in thyroid cancer incidence in the United States: review of incidence trends by socioeconomic status within the surveillance, epidemiology, and end results registry, 1980-2008. Thyroid. 2013;23(1):103-10.

4. Reis EM, Ojopi EP, Alberto FL, et al. Large-scale transcriptome analyses reveal new genetic marker candidates of head, neck, and thyroid cancer. Cancer Res. 2005;65:1693-9.

5. Kelman AS, Rathan A, Leibowitz J, Burstein DE, Haber RS. Thyroid cytology and the risk of malignancy in thyroid nodules: importance of nuclear atypia in indeterminate specimens. Thyroid. 2001;11:271-7.

6. Fugazzola L, Colombo C, Perrino M, et al. Papillary thyroid carcinoma and inflammation. Front Endocrinol. 2011;2:88.

7. Whiteside TL. Th e tumor microenvironment and its role in promoting tumor growth. Oncogene. 2008;27:5904-12.

8. Moore MM, Chua W, Charles KA, et al. Inflammation and cancer: causes and consequences. Clin Pharmacol Ther. 2010;87:504-8.

9. McMillan DC. Th e systemic inflammation-based Glasgow prognostic score: a decade of experience in patients with cancer. Cancer Treat Rev. 2013;39:534-40.

10. Azab B, Bhatt VR, Phookan J, Murukutla S, Kohn N, Terjanian T, Widmann WD. Usefulness of the neutrophil-tolymphocyte ratio in predicting short- and long-term mortality in breast cancer patients. Ann Surg Oncol. 2012;19:217-24.

11. Hanahan D, Weinberg RA. Hallmarks of cancer: the next generation. Cell. 2011;144:646-74.

12. Proctor MJ, McMillan DC, Morrison DS, et al. A derived neutrophil to lymphocyte ratio predicts survival in patients with cancer. Br J Cancer. 2012;107:695-9.

13. Kolaczkowska E, Kubes P. Neutrophil recruitment and function in health and inflammation. Nat Rev Immunol. 2013;13:159-75.

14. Ardi VC, Kupriyanova TA, Deryugina El, Quigley JP. Human neutrophils uniquely release TIMP-free MMP-9 to provide a potent catalytic stimulator of angiogenesis. Proc Natl Acad Sci USA. 2007;104:20262-7.

15. Houghton AM, Rzymkiewicz DM, Ji H, et al. Neutrophil elastase-mediated degradation of IRS-1 accelerates lung tumor growth. Nat Med. 2010;16:219-23.

16. Queen MM, Ryan RE, Holzer RG, Keller-Peck CR, Jorcyk CL. Breast cancer cells stimulate neutrophils to produce oncostatin M: potential implications for tumor progression. Cancer Res. 2005;65:8896904. 
17. Kao SC, Pavlakis N, Harvie R, et al. High blood neutrophil-to-lymphocyte ratio is an indicator of poor prognosis in malignant mesothelioma patients undergoing systemic therapy. Clin Cancer Res. 2010;16(23):5805-13.

18. Cho H, Hur HW, Kim SW, et al. Pre-treatment neutrophil to lymphocyte ratio is elevated in epithelial ovarian cancer and predicts survival after treatment. Cancer Immunol Immun. 2009;58:15-23.

19. Ubukata H, Motohashi G, Tabuchi T, Nagata H, Konishi S, Tabuchi T. Evaluations of Interferongamma/Interleukin-4 Ratio and Neutrophil/ Lymphocyte Ratio as Prognostic Indicators in Gastric Cancer Patients. J Surg Oncol. 2010;102:742-7.

20. Guthrie GJK, Charles KA, Roxburgh CSD, Horgan PG, McMillan DC, Clarke SJ. The systemic inflammation-based neutrophil-lymphocyte ratio: Experience in patients with cancer. Crit Rev Oncol Hemat. 2013;88:218-30.

21. Gomez D, Morris-Stiff G, Toogood GJ, Lodge JPA, Prasad KR. Impact of systemic inflammation on outcome following resection for intrahepatic cholangiocarcinoma. J Surg Oncol. 2008;97:513-8.

22. Wang DS, Ren C, Qiu MZ, et al. Comparison of the prognostic value of various preoperative inflammation-based factors in patients with stage III gastric cancer. Tumor Biol. 2012;33:749-56.

23. Tomita M, Shimizu T, Ayabe T, Yonei A, Onitsuka T. Preoperative Neutrophil to Lymphocyte Ratio as a Prognostic Predictor after Curative Resection for Non-small Cell Lung Cancer. Anticancer Res. 2011;31:2995-8.

24. Kocer D, Karakukcu C, Karaman H, et al. May the neutrophil/lymphocyte ratio be a predictor in the differentiation of different thyroid disorders? Asian Pac J Cancer Prev. 2015;16:3875-9.

25. Seretis $C$, Gourgiotis $S$, Gemenetzis $G$, et al. The significance of neutrophil/ lymphocyte ratio as a possible marker of underlying papillary microcarcinomas in thyroidal goiters: a pilot study. Am J Surg. 2013;205:691-6.

26. Doğan M, Soyder A. Are NLR, Rate. and MPV Values Useful in Predicting Malignancy in Follicular Neoplasia, Atypia of Undetermined Significance and Suspicious Cytology? J Ankara University Faculty Med. 2020;73(1):47-52.

27. Kim SM, Kim EH, Kim BH, et al. Association of the Preoperative Neutrophilto-lymphocyte Count Ratio and Platelet-to-Lymphocyte Count Ratio with Clinicopathological Characteristics in Patients with Papillary Thyroid Cancer. Endocrinol Metab (Seoul). 2015;30:494-501.

28. Gong W, Yang S, Yang X, et al. Blood preoperative neutrophil-to-lymphocyte ratio is correlated with TNM stage in patients with papillary thyroid cancer. Clinics. 2016;71:311-4.

\section{Figures}




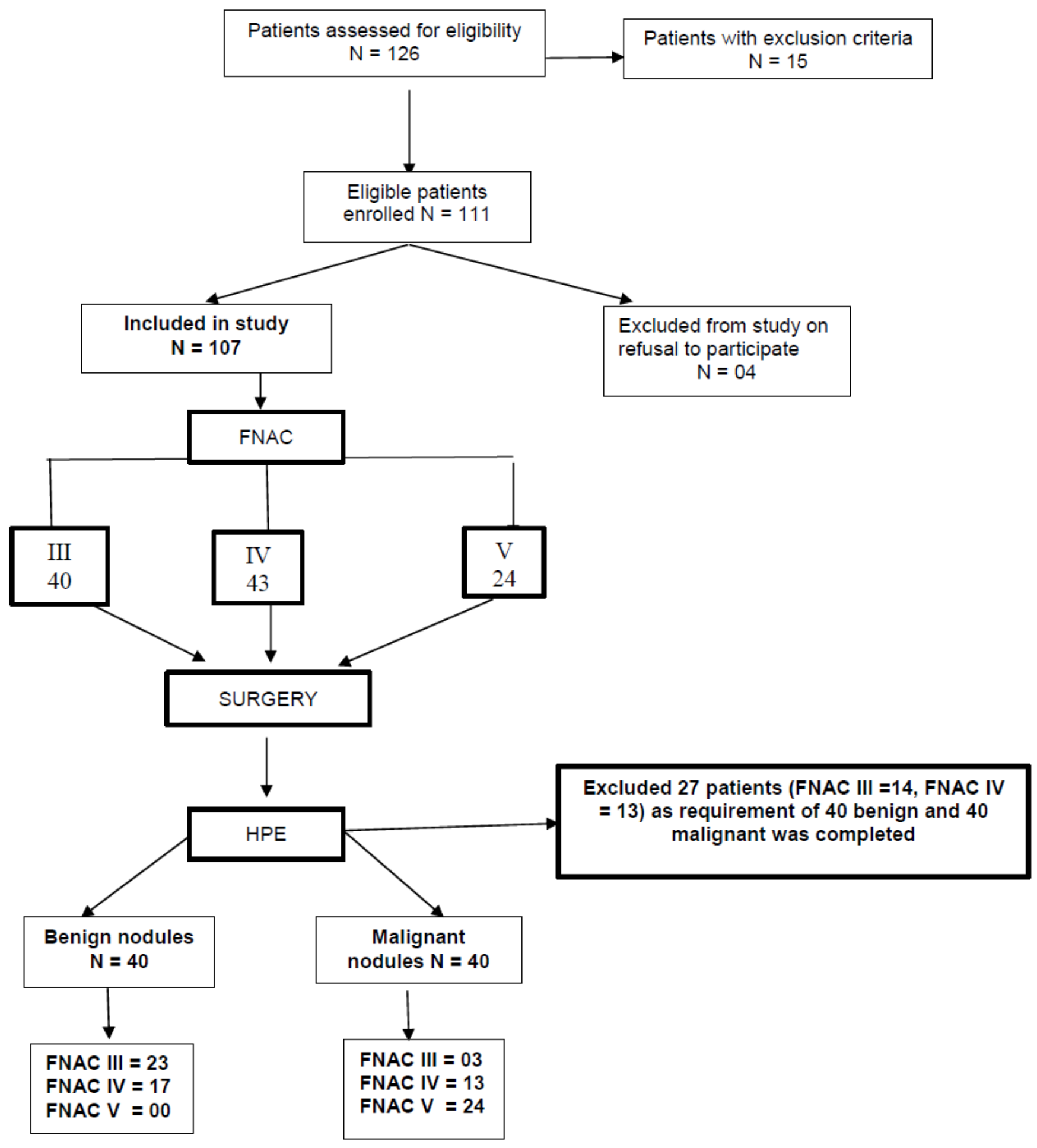

Figure 1

Flow of participants in study. 


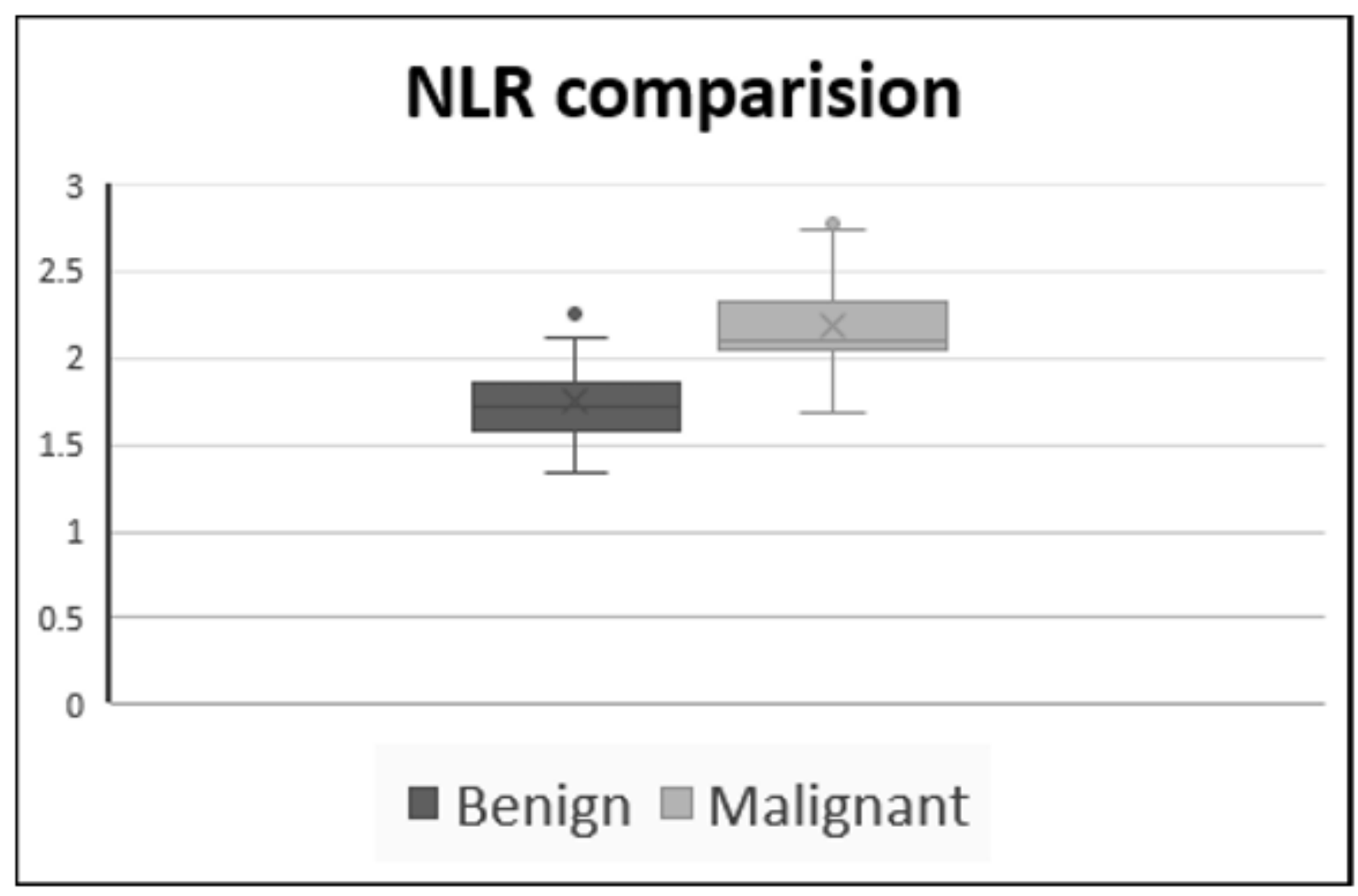

Figure 2

The Box and Whisker group comparing NLR values among both the groups
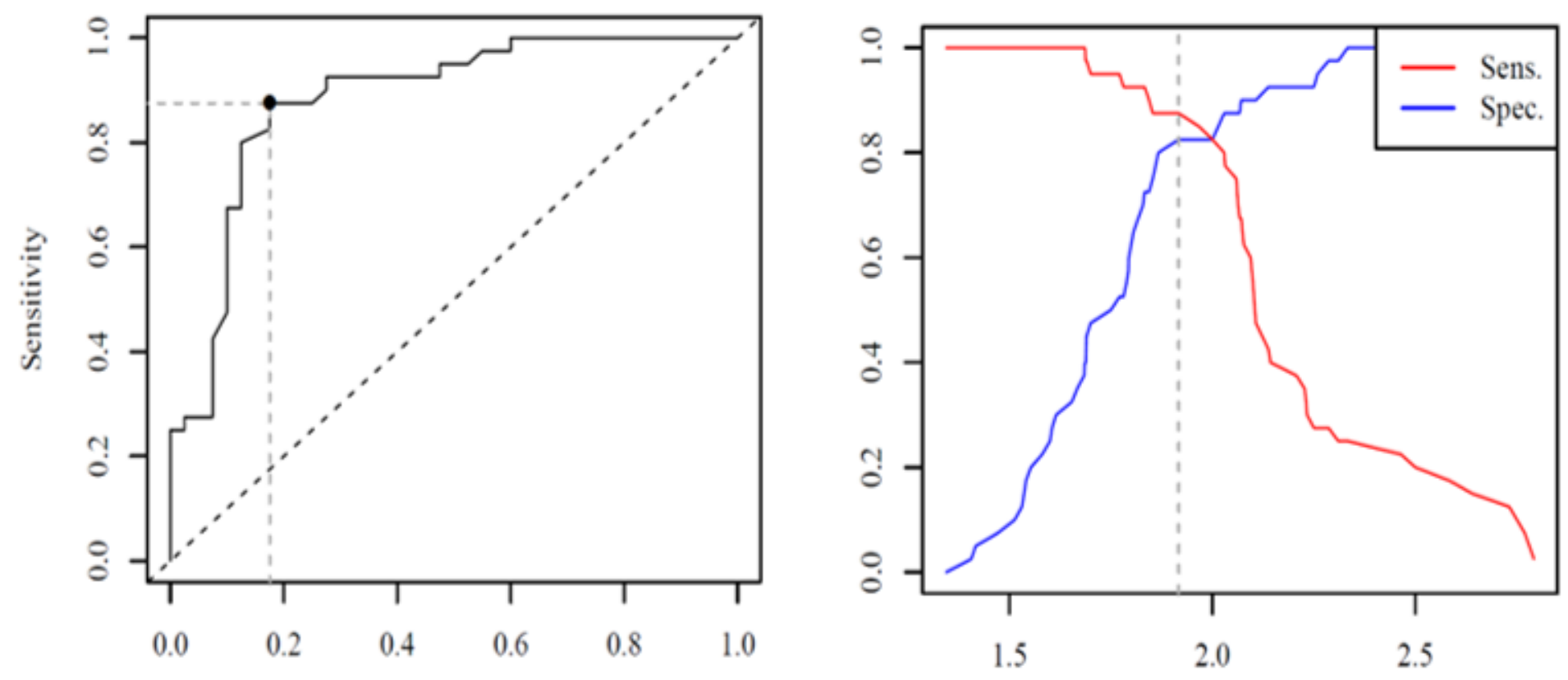

1-Specificity

Figure 3

(a): ROC curve for NLR values in both groups; (b): Sensitivity and specificity of NLR values. 

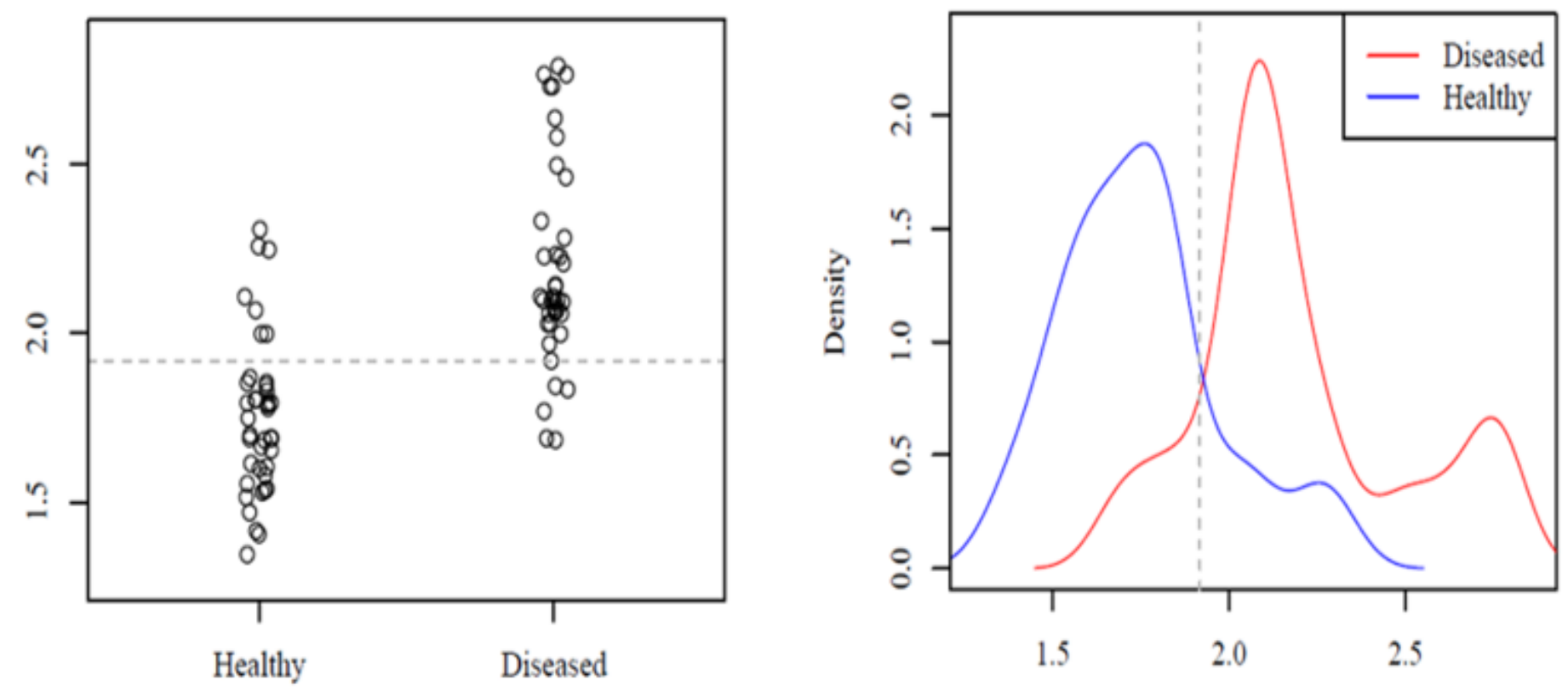

Figure 4

( $a \& b)$ : Distribution graphs for NLR values depicting best estimated cut off value for malignancy.

\section{Supplementary Files}

This is a list of supplementary files associated with this preprint. Click to download.

- STROBEStatement.docx 